\title{
An evaluation of the integration of non-traditional learning tools into a community based breast and cervical cancer education program: The witness project of Buffalo Thelma C Hurd*1, Paola Muti², Deborah O Erwin ${ }^{3}$ and Sharita Womack ${ }^{2}$
}

\author{
Address: ${ }^{1}$ Department of Surgery, Roswell Park Cancer Institute, Buffalo, New York, USA, ${ }^{2}$ Department of Cancer Prevention, State University of \\ New York at Buffalo, Buffalo, New York, USA and ${ }^{3}$ University of Arkansas for Medical Sciences, Little Rock, Arkansas, USA \\ Email: Thelma C Hurd* - thelma.hurd@roswellpark.org; Paola Muti - muti@buffalo.edu; Deborah O Erwin - erwindeboraho@uams.edu; \\ Sharita Womack - sdwomack@buffalo.edu \\ * Corresponding author
}

This article is available from: http://www.biomedcentral.com/147I-2407/3/18

(C) 2003 Hurd et al; licensee BioMed Central Ltd. This is an Open Access article: verbatim copying and redistribution of this article are permitted in all media for any purpose, provided this notice is preserved along with the article's original URL.

\begin{abstract}
Background: Breast and cervical cancer continue to represent major health challenges for African American women. among Caucasian women. The underlying reasons for this disparity are multifactorial and include lack of education and awareness of screening and early detection. Traditional educational methods have enjoyed varied success in the African American community and spawned development of novel educational approaches. Community based education programs employing a variety of educational models have been introduced. Successful programs must train and provide lay community members with the tools necessary to deliver strong educational programs.
\end{abstract}

Methods: The Witness Project is a theory-based, breast and cervical cancer educational program, delivered by African American women, that stresses the importance of early detection and screening to improve survival and teaches women how to perform breast self examination. Implementing this program in the Buffalo Witness Project of Buffalo required several modifications in the curriculum, integration of non-traditional learning tools and focused training in clinical study participation. The educational approaches utilized included repetition, modeling, building comprehension, reinforcement, hands on learning, a social story on breast health for African American women, and role play conversations about breast and cervical health and support.

Results: Incorporating non-traditional educational approaches into the Witness Project training resulted in a $79 \%$ improvement in the number of women who mastered the didactic information. A seventy-two percent study participation rate was achieved by educating the community organizations that hosted Witness Project programs about the informed consent process and study participation.

Conclusion: Incorporating non-traditional educational approaches into community outreach programs increases training success as well as community participation.

\section{Background}

Breast cancer represents a serious health problem for the
African American community. In New York State, breast cancer mortality increased $1.86 \%$ among African American 
women while breast cancer mortality in Caucasian women decreased 9.2\% between 1986 and 1995 [1]. Mammographic screening, resulting in early diagnosis, contributed significantly to this mortality decrement among Caucasian women. The disparity in mortality among African Americans is multifactorial and includes lack of education and awareness of the importance of early screening and detection, lower screening rates, advanced stage disease at diagnosis, lack of or limited access to services, delay in treatment, lack of consistent health care providers, distrust of the health care system, lack of health insurance and biology [2-8]. The educational and awareness issues are further complicated by cultural myths and beliefs that foster a fatalistic, fearful, conceptualization of breast cancer and that cross socioeconomic lines [9].

Traditional approaches to cancer education have been modeled upon white women's attitudes and belief systems and have been largely unsuccessful in the African American community due to limited cultural relevance and interaction. The requirement that effective educational program present information within an appropriate cultural context in order to gain wide community acceptance and behavior modification is a concept that is now being recognized and implemented.

The Witness Project of Buffalo is a community based, culturally sensitive breast cancer health education program, targeting African American women that is modeled after the Witness Project at the University of Arkansas. This program empowers women, through education, to take responsibility for their health and effect change in health care behaviors. The primary goals of the Witness Project of Buffalo are to increase African American women's awareness of breast self-exam (BSE), clinical breast exam and screening mammography as early detection methods for breast cancer and to improve the utilization rates of existing breast cancer screening and diagnostic programs in Erie County, New York.

Lay health advisors and witness role models form the foundation for delivery of the Witness Project to the community. They simultaneously function as educators and natural helpers and their training is designed to integrate and exploit these attributes. Described herein is an eight week training program created to help reduce breast and cervical cancer morbidity and mortality rates within the African American community. Implementation of this adult training program in Buffalo required the integration of non-traditional approaches in addition to the methods outlined in the Witness Project Training Manual. We report on the development, implementation and evaluation of these non-traditional learning tools into an adult educational program for lay educators in the Witness Project of Buffalo.

\section{Methods \\ The community}

The Buffalo metro area (population 292,000), located in Erie county New York is struggling to transition and redefine itself from a manufacturing economy to a more diverse, global oriented focus and to train its workforce to meet future needs. Since 1993-94 the minority population of western New York has increased from 12 to $13 \%$ and there are now 140,000 African Americans. Of these $109,200(78 \%)$ live in Erie County (predominately in Buffalo). The poverty rate is higher than the national average with thirty five percent of African Americans living below the poverty level (2000 Census). Churches play a dominant role as centers for community outreach. Approximately $65 \%$ of African American women in Buffalo attend church.

The African American community dates back to the Civil War since Buffalo was the last stop on the Underground Railroad. As a result, the community that has developed and evolved early on consisted of former slaves and in subsequent decades, individuals migrating from the south seeking a better life. The community is characterized by extremely well integrated and close relationships between members and remains open to novel ideas and initiatives which are critically evaluated and selective. The church continues to be the solid, influential and far reaching center for African American culture and society simultaneously. As a result, community access is primarily controlled through the church. In addition to churches, there are several other community centers serving as the basis for social focus which are both public and church affiliated centers.

\section{Witness Project program design}

Witness Project programs are delivered in the community setting (i.e. church, community center), by an educational team comprised of two witness role models and one lay health advisor, recruited from the African American community. The educational team organizes and presents grassroots outreach programs that promote early detection of breast and cervical cancer. Witness role models are breast or cervical cancer survivors who are willing to share their experiences with breast cancer, offer messages of empowerment, optimism and success as the result of early diagnosis and screening and serve as a local resource. Lay health advisors, who may or may not be cancer survivors, serve as educational and health service resources.

At a typical program, witness role models share their personal story with the audience highlighting the importance of early detection, BSE and yearly screening. Lay health 
advisors present didactic information about breast and cervical cancer screening and diagnosis and teach breast self-exam using an interactive approach. The team then answers questions and distributes educational materials to the audience. Audience members at Witness Project programs sign informed consent and complete a registration form while at the program. The registration form enables Witness Project personnel to contact program participants and arrange follow-up screening tests. All programs begin with prayer and a hymn.

\section{WRM and LHA training}

The Witness Project educational model is a belief and learning theory based intervention that incorporates the health belief model, locus of control beliefs, social learning theory and the trans theoretical model of change and the 4MAT System [10]. The training utilizes a self study approach that is supplemented with traditional learning tools i.e. slides and printed materials.

Training is initiated by having all witness role models and lay health advisors attend an actual Witness Project program on the first day. On the second day, witness role models and lay health advisors undergo a formal 8 hour, training class. Lay health advisors take a short, pre-test to assess their fund of knowledge about breast and cervical cancer prior to and following formal training. All trainees then receive an overview of the Witness Project and view a short video about the Project. Confidentiality issues and expectations, facts pertaining to breast and cervical cancer, breast self-examination (BSE), mammography and Pap tests are reviewed in detail using a slide presentation. Break out sessions are conducted by project staff for witness role models and lay health advisors respectively. Witness role models introduce themselves, tell their stories to the group and receive group feedback on their presentation. Lay health advisors learn how to perform and teach BSE. Both groups reconvene, and learn how to set up a Witness Project program.

Following the initial training session, the outreach training coordinator for the program assists witness role models in developing their stories, condensing them into short narratives, and learning and incorporating the public speaking skills required to deliver their message in a relaxed and inviting manner. Lay health advisors study the didactic information presented in the course outline, learn to teach BSE in a mentored setting and practice the delivery of their message.

\section{Description of the sample}

Participation in the Witness Project required that both witness role models and lay health advisors be articulate, comfortable speaking to people, possess a desire to provide health education, commit to an intensive training program, have a commitment to improving cancer education in the community, be willing to be available to the community and to act as a health information resource. Although the Witness Project of Buffalo provided training stipends for the initial 8 hour training to lay health advisors and witness role models, women were not told of this until they reported to the training session.

Twenty three women (9 lay health advisors, 14 witness role models) comprised the initial training group. Witness role models and lay health advisors were recruited from one author's surgical oncology practice $(\mathrm{TH})$ as well as by word of mouth and through social organizations. The professional, socioeconomic and employment experience diversity of the Witness Project staff resulted in recruitment of a diverse group of women to the project. The median age of individuals in the group was 52 years (range 33 years - 70 years). All had finished high school and $35 \%$ had completed college. All completed the initial eight hour training course.

\section{Training interventions}

The complete training process for both groups was initially expected to require approximately 2 months. All witness role models completed their training within 6-8 weekly training sessions. However, lay health advisors required a longer training period as well as the integration of non traditional learning tools into their training. The approaches used to achieve a $100 \%$ successful outcome among the witness role models and lay health advisors is described as follows.

\section{Witness role models}

The standard core teaching method worked well for the Witness Role Models. Educational contracts and videotaping presentations were the only two non-traditional tools employed for role model training. During the first two sessions, each woman shared her experiences with the role model group and each presentation was timed. At the end of the second session, role models were instructed to write their story in a two to three page narrative prior to the third session. Individuals who had difficulty writing a narrative were encouraged to write one half to one page of talking points. Individuals who had difficulty expressing their thoughts in either of these two formats met with the training coordinator in individual sessions and formulated a narrative together that accurately conveyed the role model's experience. These approaches provided a narrative that could be presented within 3-5 minutes. The stories were then read, and each critiqued for presentation and content by fellow witness role models in the group in a supportive and non threatening fashion. Major changes recommended by the group were incorporated into an individual's presentation. 
Once the presentation content was mastered, attention focused upon presentation skills and stage presence. Each witness role model signed a contract that required her practice of her presentation ten minutes per day in front of the mirror and present her talk to at least one or two individuals per week. At weekly meetings, witness role models each presented their stories and were critiqued by fellow role models on content, delivery and stage presence. This resulted in a relatively rapid assimilation of presentation skills. Finally, witness role models were videotaped and each woman as well as the group critiqued the video. This method allowed for maximal and rapid improvement in and optimization of presentation delivery.

\section{Lay health advisors}

The educational approach outlined in the training manual was followed during the first 6 weeks of our training. Weekly training sessions for lay health advisors began within three weeks of the initial training course. In order to assess the knowledge level, a short written quiz, replicating many of the items on the post training course quiz, was administered to lay health advisors. The average score on the first quiz following the initial 8 hour training course was $60 \%$. The Witness Project core curriculum establishes $80 \%$ as the minimum passing score.

Training began by teaching breast and cervical cancer facts, followed by mammography, Pap facts and BSE and utilized the self study approach outlined in the core curriculum. The training team met weekly with the lay health advisors to review the assigned information in the course outline covered during the preceding week of study. At the end of the first 6 weeks, only 11\% (2/9) of women acquired the didactic knowledge and skills necessary to present a program.

A focus group session was conducted to determine the problems faced by the students. Difficulty organizing the information, difficulty remembering the facts, discomfort with medical terms, fear of presenting the information accurately, lack of time to study, and feeling overwhelmed by the volume of information were the most common problems cited by the group. These results prompted our group to reassess the Witness Project approach to teaching didactic information and make significant adaptations to meet the specific needs of our group by introducing nontraditional learning tools as part of a concrete approach to learning.

Five educational tools that are not normally incorporated into community-based learning programs were implemented: educational contracts, vocabulary development, accessing prior knowledge, question and answer training format, and role playing.
Educational contracts required women to act on their new knowledge by encouraging learning of the information, use of the breast and cervical health information, and promoted studying through a written commitment. Each lay health advisor signed an educational contract that required her to: 1.) study the health fact and BSE packet daily, 2.) practice the lay health presentation daily and 3.) share the information with three female friends or family members weekly. Lay health advisors directly related their past experiences and knowledge about breast and cervical cancer screening and integrated them with changing behaviors and preventive health practices.

The core curriculum didactic information is presented in outline format in the training manual and proved difficult for the trainees to assimilate. To overcome this, we utilized a concrete approach to learning in which all of the information was reorganized and presented in two formats: color coded flash cards corresponding to each curriculum component which were organized into teaching modules and written and verbal question and answer formats. Each module was taught in 1-2 weeks and written quizzes were given to assess knowledge level at the end of each week. A medical vocabulary was developed that introduced the medical terms and concepts, in glossary format, used in the Witness Project curriculum. Individuals who did not achieve an $80 \%$ score were tutored in a small group or individual setting. Trainees did not progress to the next module until a passing score was achieved on the written test.

After mastering the flashcards, trainees progressed to a question and answer training format that covered the same information presented in the flashcards. Breast and mammography facts were combined and cervical and Pap facts were combined into a total of 20 questions, thus further consolidating the information. Four to five questions were assigned on a weekly basis. Questioning skills were emphasized to promote thinking, focus on information, organize responses and build comprehension.

Role playing was also integrated into this phase of our training program. At each session, trainees were paired with one person acting as the questioner and the other as the respondent. Roles were reversed and the entire process monitored by Witness Project staff. Role playing reinforced the importance of sharing breast health information with family, friends and the community and fostered creative thinking, i.e. how the information would be presented.

These approaches continued to build comprehension and confidence, focused students on organizing their responses utilizing their fund of knowledge, promoted thinking and simulated question and answer sessions at 
programs. At the beginning of each session an oral quiz was given to the trainees. This technique improved verbal skills, simulated questions that lay health advisors would encounter at programs and increased comfort with this format. All of the written and oral examinations used to test competency during our training program built on the prior week's session information. The tests became increasingly more difficult such that the final tests encompassed all of the information covered in a particular flash card or question format training module.

\section{Breast self-examination (BSE)}

BSE training required 2-3 weeks and was conducted by witness project staff who were certified BSE instructors or physicians and followed the core curriculum. The lay health advisors were paired and taught each other BSE under the supervision of trained Witness Project staff. Each person critiqued her partner's training in a constructive fashion thus creating a relaxed atmosphere that fostered continued experiential growth in teaching BSE. Correctly teaching BSE to a trained Witness Project staff member completed this module of training. A tour of the Mammography Center at Roswell Park Cancer Institute was conducted by the chief mammographer to complement and complete the BSE training. This tour enabled lay health advisors to link didactic information with practical experience.

\section{Confidentiality issues and informed consent}

The Witness Project core curriculum addressed confidentiality by stressing the importance of respecting individual privacy and not circulating information gained through encounters with individuals attending a Witness Project program but lacked specific training guidelines in this area. Our program requires participants to sign informed consent so that Witness project personnel can contact them at later time periods, provide informational resources and evaluate program effectiveness. The integration of informed consent training is a novel and non-traditional approach to community-based education programs that enabled us to interact with the community more effectively and enhance study participation.

Addressing informed consent issues required intervention at the trainer level (lay health advisors, witness role models) as well as the community based organization level. All lay health advisors and witness role models received formal training about the informed consent process from two individuals (SW, TH) who had experience in this area. The rationale, historical significance, ethics, regulatory requirements and oversight components of informed consent as well as approaches for obtaining informed consent were taught in two training sessions. During the second session, participants practiced obtaining informed consent from each other (using the consent form for the Wit- ness Project of Buffalo) under the supervision of Witness Project staff. Trainees critiqued each others' presentations. This approach alleviated trepidation regarding the consent process and enabled the witness role models and lay health advisors to obtain informed consent from program audience members in a relaxed, controlled and informed fashion thus allaying audience fears about project participation.

To establish comfort and trust with the community-based organization hosting our program, the outreach coordinator met with the program host and introduced and reviewed the consent form and procedure and answered all questions. A copy of the consent was given to the host organization for review and discussion of subsequent questions or issues, if needed, prior to the scheduled program.

\section{Results}

The standard training approach for didactic information outlined in the training manual required 6 weeks and was successful in only $2 / 9(22 \%)$ of our lay health advisors. Incorporating non-traditional learning tools into our curriculum resulted in a dramatic improvement in the time required for the remaining seven lay health advisors to master the didactic information. Two of the seven lay health advisors mastered the didactic information within 6 weeks of incorporating non-traditional learning tools, completed their practical training and began conducting programs after a total of 10 weeks of instruction utilizing the non-traditional educational methods. Four (57\%) required 6-8 weeks to assimilate the didactic information and an additional 4 weeks of practical training to conduct a program. Fourteen percent of the lay health advisors required 16 weeks to master the didactic material and an additional 8 weeks to master the presentation skills.

The integration of structured training about informed consent and confidentiality is a novel approach in community based educational programs. Structured training for witness role models and lay health advisors enabled our program to overcome the barriers to consent and study participation. Many of the issues and reservation relating to research studies and informed consent that our witness role models and lay health advisors expressed were openly discussed. This enabled the women to convey information to program participants using a forthright and informed approach that allayed many of the audience members trepidation. An open and transparent discussion of the clinical study and informed consent with the sponsoring host and church leadership reduced and in virtually all cases eliminated concerns about this aspect of the Witness Project of Buffalo. This approach resulted in a consent rate of $40 \%$ for the initial six programs, $61 \%$ for programs $7-10$ and $100 \%$ by the thirteenth program. We 
have now conducted over 40 programs with an overall study consent rate of $79 \%$.

\section{Discussion}

Community based cancer education requires interventions at many levels that address the fundamental, causative issues contributing to the myriad of health disparities, i.e. access, health insurance, transportation, MD referral, lack of time, fear, fatalism and lack of knowledge. The community's perception of specific health issues has a major role in determining response to educational interventions. One study evaluated community perception of health status in one geographic area which was classified as one of the least healthy areas of the region. However, $80 \%$ of people surveyed described their health as good to excellent despite the fact that $68 \%$ had never had mammograms, $40 \%$ had not had a Pap smear within the preceding year, only $20 \%$ had mammography the preceding year and 50\% felt their neighborhood environment was unsafe. The majority of respondents felt that health education was a top priority in their community [11]. Also, many African Americans have a false perception that the incidence of cancer is lower in African Americans than in Caucasians [12].

A successful community based cancer educational programs require several components: educational programs, appropriate training, outreach individuals, strong relationships with the public and private medical providers and navigational services. As confirmed in this report, lay health advisors can be a highly effective component of the cancer educational program in their role as trained community educators and liaisons between the community and medical providers. They commonly function in the community as natural helpers, (lay people from whom others seek emotional support, aid and advice), possess environmental familiarity and are highly respected and trusted $[13,14]$. They can address initial health concerns often bridging the chasm between the doctor or health care provider and the patient. Perhaps their most important role is addressing health care issues within the social network of the community which can translate into increased trust in the health care system and empowerment of community members to adopt proactive rather than reactive behaviors relative to personal health. Salber et al [15] have identified additional functions of the lay health advisor that include strengthening professionals' ties with the community, channeling knowledge to and from the community, bidirectional education of community members on the role and function of the health care system and of professionals about the lay advising system, informing the community about available resources, improving the helper's role in helping others and helping the community to better cope with health care problems.
Lay health advisors also mobilize community resources to sustain and support the health care system [16].

Many terms have been used to describe lay health advisors (natural helpers, facilitators, community health workers). Lay health workers comprise an increasing number of the health care agency workforce. In northern California, 41\% of 197 community based organizations employed or planned to employ community health workers to reach out to their target communities [16]. The Pew Health Professions Committee defines them as "community members who work almost exclusively in community settings and who serve as connectors between health care consumers and providers to promote health among groups that have traditionally lacked access to adequate care" [16]. Their roles in health delivery are varied and span the spectrum from volunteers offering emotional support to full time health care employees providing medical information at the lay level. Some have argued that training community individuals to deliver health information destroys their ability to function as natural helpers [17]. In the North Carolina Save Our Sisters Project, lay health advisors are the functional unit of one outreach initiative and are trained as natural helpers. They assist individuals in their community with needs that are difficult for medical professionals to address, negotiate with professionals for health system support and mobilize community association resources to sustain support from the health care system $[13,18,19]$. The environment in which they work can vary from small group to individual interactions in intimate as well as depersonalized environments [20,21]. In the Arkansas Witness Project, lay health advisors have an integrated role as both natural helpers and educators [22].

Training for lay health advisors must carefully integrate the scientific aspects of the information that they disseminate to the community with maintaining and strengthening the natural helper aspects of their position. The Witness Project curriculum has incorporated multiple learning theories (PRECEDE, 4MAT, health belief model, locus of control, transtheorectical theory, community empowerment and PEN-3) into the design of the educational program [23]. However the witness role model and lay health advisor training module is based on the 4MAT system which addresses the four distinct adult learning types as well as right and left brain and integrated learners. Briefly, type 1 learners perceive by sensing/feeling and process by watching/reflecting. They learn by connecting past and present experiences and then examining the connection. Type 2 learners perceive by thinking/reasoning and processes by watching/reflecting and prefer informational learning. They learn by imaging the concept and then defining it with facts and information eg. Mastering and conceptualizing information regarding breast selfexam and mammography facts. Type 3 learners perceive 
by thinking/reasoning and process by doing/trying. They focus on application and prefer using the coaching method. They test concept implications and then elaborate and reconstruct the defined concepts (practicing BSE). Type 4 learners perceive by sensing/feeling and process by doing/trying. They learn by personal adaptation and self discovery and evaluate and modify concepts to integrate new connections [22].

In the Witness Project core curriculum, lay health advisors develop the concepts and skills to provide health education by being taught the facts about breast and cervical cancer. This aspect of the training program is heavily weighted toward the type 2 learner. Our lay health advisors had great difficulty with this approach. Therefore, we incorporated the non-traditional learning tools described in this paper into our curriculum to provide heavier emphasis on type 3 and type 4 learning styles.

The educational contract served as tangible commitment to learning. An individualized learning approach wherein lay health advisors progressed through the educational process at their own pace provided a relaxed academic atmosphere. Objective evaluation of their training necessitated adherence to standard approaches designed for the type 2 learners. However, by progressively increasing test difficulty and integrating both written and verbal testing formats, test taking anxiety was almost completely eliminated. Furthermore, the verbal testing formats were complemented by re-presenting the didactic information in a question and answer format which further alleviated anxiety and increased confidence. With this approach, all lay health advisor candidates were able to comfortably address questions posed to them during Witness Project program presentations.

Witness role model training proved less challenging than for lay health advisors. Verbal and written recapitulation of their experiences enabled role models to create, reflect upon and analyze their experiences with breast or cervical cancer. Sharing their presentations with other role models and receiving thoughtful criticism and praise increased self confidence and performance. The relevance and usefulness of their experience were analyzed through group practice sessions which included critiquing of presentations for delivery and content. Anxiety about public speaking was almost completely eliminated. More importantly, the importance and benefit of sharing their personal story with strangers was reinforced. As the training process continued, they developed original applications by enhancing their stories or began creating new stories based upon different aspects of their illness, eg. screening, diagnosis, treatment, reconstruction, family and spiritual support. This particular phase (creating new stories) of their development did not generally occur until the witness role models had been giving programs 6 months.

One of the most important and novel aspects of our training focused upon informed consent and study participation issues. There is little published data regarding the issue of informed consent in community-based programs. Prior community outreach initiatives have dealt with informed consent issues by obtaining consent in general terms, utilizing university coverage, obtaining verbal consent or removing identifiers form data to eliminate the need for informed consent. Our program is unique in two ways: 1.) lay members of the community receive formal training about informed consent and are taught how to obtain it and 2.) the informed consent process is specifically reviewed with the hosting community organization, an approach that has not been previously reported.

Our primary goal was to educate our role models and lay health advisors about the informed consent process, allay fears related to participating in a clinical study and give them the tools to repeat this same process within the community. This concept was perpetrated at the individual as well as the organizational level (program host). By directly addressing fears and anxieties about clinical study participation at the individual and organizational level, the issues of informed consent were minimized.

We have presented a detailed description of traditional and non traditional educational methods employed to train lay health advisors in a community based breast and cervical cancer educational program. Our educational approach was tailored to the learning needs of the lay health advisors and witness role models and differs to some extent from prior published studies in that rigorous objective evaluation of learning has been infrequently used. Others have used quantitative methods but have relied more on subjective knowledge assessment and measured effectiveness by the number of community members reached $[18,24-26]$. As community health based education continues to proliferate, it is imperative that more attention be directed to the training process and objective evaluation of this process. While it is essential to be able to effectively transfer information to the community at large it is imperative that the educational quality of the product that a training program produces (lay health advisor, witness role model) be maximized through well integrated multifaceted approaches.

\section{Conclusions}

The incorporation of non traditional learning approaches into community based educational training curriculums enhances training success and increases community participation. 


\section{Competing interests}

None declared

\section{Authors contributions}

Drs. Hurd, Muti and Erwin participated in draft preparation. Drs. Hurd and Womack designed the study. The Witness Project of Arkansas was initiated and designed by Dr. Erwin. All authors have read and approved the manuscript.

\section{Acknowledgements}

This work was funded by a grant from the Susan G. Komen Foundation. Additional support was provided by Roswell Park Cancer Institute. We would like to thank Bettina Watson and Kimberly Trammell for their assistance in this project.

\section{References}

I. Canto: CA Journal for Clinicians 200I.

2. Heimann R, Ferguson D, Powers C, Suri D, Weichselbaum RR and Hellman S: Race and clinical outcome in breast cancer in a series with long term follow-up evaluation J Clin Onc 1997, I 5(6):2329-2337.

3. Chen F, Trapido EJ and Davis K: Differences in stage at presentation of breast and gynecologic cancers among whites, blacks and hispanics Cancer 1994, 73:2838-2842.

4. Newman LA, Mason J, Cote D, Vin Y, Carolin K, Bouwman D and Colditz GA: African American ethnicity, socioeconomic status and breast cancer survival Cancer 2002, 94:2844-2855.

5. McWhorter WP and Mayer W]: Black/white differences in type of initial breast cancer treatment and implications for survival $A m$ J Public Health 1987, 77(12): I515-1517.

6. Whitman W, Ansell D, Lacey L, Chen E, Ebie N, Dell J and Phillips CW: Patterns of breast and cervical cancer screening at three public health centers in an inner city urban area $A m \mathrm{~J}$ Pub Health I99I, 8I(I 2): I65I-I653.

7. Wells BL and Horm JW: Stage at diagnosis in breast cancer: Race and socioeconomic factors Am J Pub Health 1992, 82(1 0): I 383-1385.

8. Farley TA and Flannery JT: Late stage diagnosis of breast cancer in women of lower socioeconomic status: Public health implications Am J Pub Health 1989, 79( I I): I508-I5I 2.

9. Phillips J and Smith ED: Breast cancer control and african american women: A review Can Invest 200I, I 9(3):273-280.

10. Erwin D, Spatz TS, Stotts RC, Hollenberg JA and Deloney LA: Increasing mammography and breast self-examination in African American women using the witness project model J Can Ed 1996, I I(4): 10-215.

II. Stringfield YN: Perceptions of one african american community about its' health, health status and safety ABNFJ 2000 , I I(3):7|-74.

12. Lowe JI, Barg FK and Bernstein M: Educating african americans about cancer prevention and detection: $A$ review of the literature Soc Work Health Care 1995, 2 I (4): I 7-36.

13. Eng E: Natural helping functions of lay health advisors in breast cancer education $\mathrm{Br}$ Can Res Treat 1995, 35:23-29.

14. Eng E, Parker E and Harlan C: Lay health advisor intervention strategies: A continuum from natural helping to paraprofessional helping Health Education and Behavior 1997, 249:4I3-4I7.

15. Salber E: The lay advisor as a community health resource J Health Politics, Policy Law 1979, 3:469-478.

16. Love Jl, Barg FK and Bernstein MW: Educating African Americans about cancer prevention and detection: A review of the literature Social Work in Health Care 1997, 2 I (4): I7-36.

17. Salber E: The role of the health facilitator in community health education J Comm Health 1976, 2: I5-20.

18. Eng E: The save our sisters project: A social network strategy for reaching rural black women Cancer 1993, 72:107|-1077.

19. Eng $E$ and Smith J: Natural helping functions of lay health advisors in breast cancer education $\mathrm{Br}$ Can Res and Treat 1995, 35:2329.
20. Robinson KD, Kimmel EA and Yasco JM: Reaching out to the african american community through innovative strategies ONF 1995, 22(9): 1383-1391.

21. Earp JL and Flax VL: What lay health advisors do Can Pract 1999, 7(1): $16-21$.

22. Erwin D, Spatz TS and Turturro CL: Development of an africanamerican role model intervention to increase breast selfexamination and mammography $J$ Can Ed 1992, 7(4):31 I-319.

23. NCl: Theory at a Glance [httm://nci.nih.gov].

24. Milligan S, Maryland $P$, Ziegler $\mathrm{H}$ and Ward $A$ : Natural helpers as street health workers among the urban black elderly Gerontologist 1987, 27(6):7|2-7|5.

25. Jackson EJ and Parks CP: Recruitment and training issues from selected lay health advisor programs among African Americans: A 20 year perspective J Health Ed and Behav 1977, 24(4):4|8-43|.

26. Earp JL, Viadro $\mathrm{Cl}$, Vincus A, Alpeter M, Flax V, Mayne L and Eng E: Lay health advisors: A strategy for getting the word out about breast cancer Health Ed and Behav 1997, 24(4):432-45 I.

\section{Pre-publication history}

The pre-publication history for this paper can be accessed here:

\section{http://www.biomedcentral.com/1471-2407/3/18/prepub}

Publish with Biomed Central and every scientist can read your work free of charge

"BioMed Central will be the most significant development for disseminating the results of biomedical research in our lifetime. "

Sir Paul Nurse, Cancer Research UK

Your research papers will be:

- available free of charge to the entire biomedical community

- peer reviewed and published immediately upon acceptance

- cited in PubMed and archived on PubMed Central

- yours - you keep the copyright
BiolMedcentral 